\title{
Correction to: Relationship between plasma exposure of zolpidem and CYP2D6 genotype in healthy Korean subjects
}

\author{
Eui Hyun Jung ${ }^{1}$ Choong-Min Lee ${ }^{1} \cdot$ Ji-Yeong Byeon ${ }^{1} \cdot$ Hyo-Bin Shin ${ }^{1} \cdot$ \\ Kyung-Yul Oh ${ }^{1}$ - Chang-Keun Cho ${ }^{1}$ Chang Woo Lim ${ }^{1}$ - Choon-Gon Jang ${ }^{1}$. \\ Seok-Yong Lee ${ }^{1} \mathbb{D} \cdot$ Yun Jeong Lee ${ }^{2}$
}

Published online: 30 January 2021

(c) The Pharmaceutical Society of Korea 2021

\section{Correction to: Arch. Pharm. Res. (2020) 43:976-981 \\ https://doi.org/10.1007/s12272-020-01250-1}

Unfortunately, there is an error in the fund number, so we correct it as follows.

Acknowledgements This research was supported by the Basic Science Research Program through the National Research Foundation of Korea (NRF) funded by the Ministry of Science, ICT, \& Future Planning (NRF2016R1A2B4007381 and NRF-2019R1A2C1004582).

Publisher's Note Springer Nature remains neutral with regard to jurisdictional claims in published maps and institutional affiliations.

The original article can be found online at https://doi. org/10.1007/s12272-020-01250-1.

Seok-Yong Lee sylee@skku.ac.kr

$\bowtie$ Yun Jeong Lee yunlee@dankook.ac.kr

1 School of Pharmacy, Sungkyunkwan University, Suwon 16419, Republic of Korea

2 College of Pharmacy, Dankook University, Cheonan 31116, Republic of Korea 